\title{
Contaminação mercurial em pescado capturado na lagoa Rodrigo de Freitas - Rio de Janeiro, Brasil
}

\author{
Mercuric contamination of the fish inside \\ Rodrigo de Freitas lagoon - Rio de Janeiro, Brazil
}

\author{
Micheli da Silva Ferreira, ${ }^{*}$ Eliane Teixeira Mársico, ${ }^{*}$ Sérgio Carmona de São Clemente, ${ }^{* *}$ Renata Jurema Medeiros ${ }^{*}$
}

\begin{abstract}
Resumo
O objetivo deste estudo foi avaliar o grau de contaminação mercurial em alguns representantes da biota aquática deste ecossistema. Foram analisados 24 exemplares de acará (Geophagus brasiliensis), 18 de tainha (Mugil sp.), 18 de robalo (Centropomus sp.), 10 de siris (Callinectes sp.) e 10 pools de cracas (Balanus sp.). Para a determinação da concentração de mercúrio total, utilizou-se o analisador Bacharach Coleman, modelo MAS-50 B, baseado na técnica de espectrofotometria de absorção atômica por arraste de vapor a frio. O tratamento constou de uma análise descritiva simples e comparação das médias de concentração de $\mathrm{Hg}$ Total obtidos em cada porção estudada por análise de variância e pelo teste não-paramétrico de Friedman, com objetivo de comparar a magnitude das variações. Outra análise estatística utilizada para interpretação dos resultados foi o Coeficiente de Correlação de Pearson, onde foi medido o grau de correlação entre duas variáveis. Os teores médios encontrados foram $0,015 \mathrm{mg} \cdot \mathrm{g}^{-1}(0,001-0,105)$ nos exemplares de acará; $0,011 \mathrm{mg} \cdot \mathrm{g}^{-1}(0,002-0,063)$ nas tainhas; 0,025 $\mathrm{mg} \cdot \mathrm{g}^{-1}(0,015-0,041)$ nos robalos; $0,023 \mathrm{mg} \cdot \mathrm{g}^{-1}(0,012-0,056)$ nos siris; e $0,057 \mathrm{mg} \cdot \mathrm{g}^{-1}(0,042-0,075)$ nas cracas. Não foi encontrada correlação entre o teor de $\mathrm{Hg}$ e tamanho e/ou peso das amostras em nenhum organismo estudado. Embora os valores obtidos estejam abaixo do limite máximo permitido pela legislação brasileira, de 0,5 ppm e 1,0 ppm para predadores, é de extrema importância a monitorização dos teores de Hg na lagoa. Desta forma, os resultados servem de subsídios para uma ação dos órgãos da saúde pública quanto à liberação ou não do consumo de pescado deste ecossistema.
\end{abstract}

Palavras-chave: mercúrio, lagoa Rodrigo de Freitas, peixes, cracas, siris.

\begin{abstract}
The objective of this study was to evaluate the degree of mercury contamination in some organisms from this ecosystem. As sampling, it had been used 24 units of "acará" (Geophagus brasiliensis), 18 of "tainha" (Mugil sp.), 18 of "robalo" (Centropomus sp.), 10 of crabs (Callinectes sp.) and 10 pools of barnacles (Balanus sp.). The analytical methodology for the determination of the total mercury concentration was using Bacharach Coleman Model MAS-50B employing cold vapor atomic absorption spectrometry, which results are presented in micrograms of mercury per grams of sample $\left(\mu \mathrm{g} \cdot \mathrm{g}^{-1}\right)$. The statistical treatment of the results consisted of a simple descriptive analysis and comparison of concentration of Total $\mathrm{Hg}$ in each portion studied by analysis of variance and by Friedman test with objective of compare the magnitude of the variations. The coefficient of correlation between the variables was measured as well. The mean values obtained were $0.015 \mathrm{mg} \cdot \mathrm{g}^{-1}(0.001-0.105)$ in "acará"; $0.011 \mathrm{mg} \cdot \mathrm{g}^{-1}$ (0.002-0.063) in "tainha"; $0.025 \mathrm{mg} . \mathrm{g}^{-1}(0.015-0.041)$ in "robalo"; $0.023 \mathrm{mg} \cdot \mathrm{g}^{-1}(0.012-0.056)$ in crabs; and $0.057 \mathrm{mg} \cdot \mathrm{g}^{-1}(0.042-$ $0.075)$ in barnacle. There was not correlation between size/weight and the mercury concentration in neither studied organisms. Although the total mercury determined was bellow the allowed limit for human consumption established by Brazilian legislation, of $0,5 \mathrm{mg} \cdot \mathrm{g}^{-1}$ and $1,0 \mathrm{mg} \cdot \mathrm{g}^{-1}$ to predators fish it is so much important to monitoring mercury values in the studied lagoon. Consequently, the results can supply an action of the public health agencies related to eating or not fishes from this ecosystem.
\end{abstract}

Keywords: mercury, Rodrigo de Freitas Lagoon, fishes, barnacles, crabs.

\section{Introdução}

A poluição aquática é um dos tipos mais preocupantes de poluição uma vez que resíduos de metais pesados não são degradáveis e tendem a se acumular em organismos vivos, resultando em diversas conseqüências para a saúde humana.

O mercúrio é um dos principais contaminantes do meio marinho e, independentemente da sua forma química, a maior

\footnotetext{
* Programa de Pós-Graduação em Medicina Veterinária - Higiene Veterinária e Processamento Tecnológico de Produtos de Origem Animal -Faculdade de Veterinária, Universidade Federal Fluminense. E-mail: micheliferreira@hotmail.com

** Departamento de Tecnologia dos Alimentos. Faculdade de Veterinária, Universidade Federal Fluminense. Rua Vital Brazil Filho, 64. Niterói/RJ. 24.230-340. Brasil.

Autor para correspondência: Eliane Teixeira Mársico. E-mail: elianee@vm.uff.br.
} 
parte se concentrará no sedimento onde será formado o metilmercúrio, forma mais tóxica do mercúrio e facilmente absorvida pela biota aquática. Os alimentos constituem a principal fonte de contaminação para pessoas que não possuem exposições ocupacionais, com destaque para 0 pescado e seus derivados, que merecem especial atenção no contexto da saúde pública em função do crescimento no consumo desta fonte protéica (WHO, 1976).

A monitorização do mercúrio em diversos compartimentos ambientais é de fundamental importância, com a finalidade prevenir a emergência de risco sanitário pela exposição humana a concentrações excessivas de mercúrio pelo consumo de pescado, bem como avaliar a possibilidade de sedimentos tornarem-se fontes potenciais de contaminação para os seres vivos, o que poderá resultar em graves episódios de intoxicação mercurial.

$\mathrm{Na}$ cidade do Rio de Janeiro, a lagoa Rodrigo de Freitas, conhecida mundialmente e considerada um cartão-postal da cidade, durante os últimos anos acumulou episódios de caráter ambiental lamentáveis, com prejuízos consideráveis para sua economia e, principalmente, para a biota aquática deste ecossistema. Em estudo sobre indicações para a administração sustentável da pesca na lagoa Rodrigo de Freitas, Ferreira Junior et al. (2003) afirmaram que este ecossistema poderia produzir cerca de 720 toneladas de pescado de boa qualidade, sem mortandade, gerando, no mínimo, 250 empregos diretos e arrecadando mais de sete milhões de reais por ano, através de uma administração onde a atividade pesqueira seria o foco principal. Essa estimativa foi realizada a partir do total capturado no ano 2000.

Portanto, o objetivo geral deste estudo foi avaliar o grau de contaminação mercurial em alguns representantes da biota aquática deste ecossistema como peixes, cracas e siris. Os resultados servirão de base para possíveis interferências do poder público na lagoa Rodrigo de Freitas, no que concerne à área de saúde pública, e ainda fornecerá conhecimentos importantes para a comunidade científica relacionada com a vigilância sanitária e a ciência de alimentos.

\section{Material e métodos}

A análise de $\mathrm{Hg}$ Total foi realizada em 61 exemplares de peixes, dentre os quais 24 acarás (Geophagus brasiliensis), 18 tainhas (Mugil sp.) e 18 robalos (Centropomus sp.), e 10 exemplares de siris (Callinesctes $\mathrm{sp}$.), obtidos antes da distribuição ao comércio. Também foram coletadas cracas (Balanus sp.), desprendendo-as das bóias de isopor, com o auxílio de uma espátula em número suficiente para perfazer $4 \mathrm{~g}$ por amostra de parte mole, totalizando 10 amostras contendo vários exemplares, em função do seu diminuto conteúdo.

Os exemplares utilizados neste estudo foram adquiridos inteiros na colônia de pescadores Z-13 da lagoa Rodrigo de Freitas durante o período compreendido entre março de 2004 a fevereiro de 2006. Os peixes, siris e cracas foram transportados em recipiente isotérmico contendo gelo para o Laboratório de Controle Físico-Químico da Faculdade de Veterinária da Universidade Federal Fluminense. Os siris foram encaminhados vivos em recipientes com água para 0 mesmo laboratório, onde foram dessensibilizados no gelo.
As amostras foram estocadas em freezer comercial comum a $-25^{\circ} \mathrm{C}$ e devidamente identificadas. No caso dos peixes, a identificação foi realizada de acordo com a espécie envolvida, seguida da primeira letra desta e de números arábicos, correspondente ao número da análise, anotando-se também o dia da coleta. Os siris foram identificados com números arábicos, de 1 a 10.

Com relação às cracas, após serem abertas com auxílio de um pequeno martelo, o conteúdo (parte mole) de cada organismo foi retirado e estocado sob as mesmas condições em quantidade individual de $4 \mathrm{~g}$, totalizando 10 amostras com cerca de 15 a 20 exemplares, e também identificadas com números arábicos de 1 a 10.

Anteriormente a cada análise, as amostras de peixes e siris foram descongeladas lentamente em geladeira comercial comum a $5^{\circ} \mathrm{C}$. Após o descongelamento, estes foram mensurados com auxílio de uma régua, pesados em balança analítica de precisão. Dos exemplares de peixes foram obtidos peso e comprimento standard, seguido da retirada de porções da musculatura de cada exemplar em quantidade suficiente para a realização das análises ( $8 \mathrm{~g}$ de cada exemplar). No caso dos siris, obteve-se a largura da carapaça de cada exemplar e, com auxílio de um martelo, rompeu-se o exoesqueleto dos siris e, com uma pinça, foi retirado o tecido muscular em porções de $8 \mathrm{~g}$ de cada exemplar. As cracas, já separadas por alíquotas de $4 \mathrm{~g}$, foram descongeladas no momento da análise.

Para a determinação da concentração de mercúrio total, as amostras foram digeridas com uma mistura de ácidos sulfúrico e nítrico concentrados $\left(\mathrm{H}_{2} \mathrm{SO}_{4}+\mathrm{HNO}_{3}\right)$ em diferentes etapas de aquecimento, em triplicata e, o mercúrio total foi determinado por espectrofotometria de absorção atômica vapor frio com obtenção dos resultados em microgramas de mercúrio por gramas da amostra $\left(\mu \mathrm{g} \cdot \mathrm{g}^{-1}\right)$ tendo como base a técnica descrita por Deitz, Sell e Bristol (1973), utilizando o analisador Bacharach Coleman, modelo MAS-50 B.

Com objetivo de validar a técnica utilizada na determinação do Hg-total foi realizado um exercício de intercalibração em conjunto com dois laboratórios pertencentes ao Centro de Tecnologia Mineral (CETEM). Para esta finalidade uma amostra do peixe da espécie Xiphias gladius, espécie predadora predisposta a apresentar altos teores de mercúrio, já analisada junto à Universidade Federal Fluminense, foi analisada por duas técnicas distintas. Uma técnica utilizou um espectrômetro de absorção atômica portátil modelo RA-915+ acoplado a um aparelho de pirólise para sólidos Lumex modelo RP-91C. A outra utilizou a técnica de EAA-VF, sendo a digestão efetuada com uma mistura de ácido perclórico, ácido nítrico e ácido sulfúrico. O valor obtido para a mesma amostra de peixe nos três laboratórios demonstrou segurança nos resultados visto que não foi evidenciada diferença estatisticamente significativa ao nível de $1 \%$ de probabilidade.

O tratamento estatístico dos resultados constou de uma análise descritiva simples e comparação das médias de concentração de Hg Total de cada porção estudada por análise de variância (ANOVA) e pelo teste não paramétrico de Friedman com objetivo de comparar a magnitude das variações. Utilizouse também o Coeficiente de Correlação de Pearson, onde foi medido o grau de correlação entre as variáveis. O programa computacional utilizado foi o Bio Stat 2,0 (Ayres et al., 2000). 


\section{Resultados e discussão}

Os resultados de $\mathrm{Hg}$ total podem ser visualizados na Tabela 1, onde são verificados os valores mínimo e máximo, as médias e os respectivos desvios padrão. Os teores médios e os desvios-padrão de cada organismo podem ser observados, individualmente, na Figura 1.

Considerando as três espécies de peixes estudadas (acará, tainha e robalo), verificou-se que o maior valor médio de $\mathrm{Hg}$ total foi observado nas amostras de robalo $\left(0,025 \mathrm{mg} \cdot \mathrm{g}^{-1}\right)$ e o menor valor nas amostras de tainha $\left(0,011 \mathrm{mg} \cdot \mathrm{g}^{-1}\right)$. Por análise de variância foi possível verificar que não houve diferença significativa $(P>0,05)$ entre os teores médios de $\mathrm{Hg}$ total nos exemplares de acará e tainha. Porém, entre os exemplares de acará e robalo, e tainha e robalo, houve uma diferença entre a concentração de $\mathrm{Hg}$ total $(P<0,05)$. Com relação a uma possível correlação entre o peso e o comprimento dos exemplares dos peixes e a concentração de $\mathrm{Hg}$ total, verificouse que tal relação não foi evidenciada para nenhuma espécie com valores de $R^{2}$ bastante baixos $\left(R^{2}=0,001-0,31\right)$. Entretanto, o maior valor individual foi observado em um exemplar de acará $\left(0,105 \mathrm{mg} \cdot \mathrm{g}^{-1}\right)$ e, o menor valor, de 0,001 $\mathrm{mg} \cdot \mathrm{g}^{-1}$ também em uma amostra da mesma espécie, caracterizando uma ampla dispersão desse valor.

Nos 10 exemplares de siris (Callinectes sp.) o teor médio de $\mathrm{Hg}$ total observado foi de $0,023 \pm 0,013 \mathrm{mg} \cdot \mathrm{g}^{-1}$. Não foi observada correlação entre peso dos siris e teor de $\mathrm{Hg}$ $\left(R^{2}=0,08\right)$ nem entre tamanho dos siris (largura da carapaça) e o teor de $\mathrm{Hg}\left(\mathrm{R}_{2}=0,02\right)$.

Com relação às cracas (Balanus $\mathrm{sp}$.) representante filtrador estudado, constatou-se maior teor médio de $\mathrm{Hg}$ total que os siris, e que existe uma diferença estatisticamente significativa $(P<0,05)$ entre a concentração deste metal nestas duas espécies de pescado.

Tabela 1: Concentração média de $\mathrm{Hg}$ total dos organismos estudados oriundos da lagoa Rodrigo de Freitas-RJ obtidos entre março de 2004 e fevereiro de 2006, com respectivos hábitos alimentares, valores mínimos e máximos, médias e desviospadrão

\begin{tabular}{cccccc}
\hline \hline Organismos & $\mathrm{n}$ & $\begin{array}{c}\text { Hábito } \\
\text { alimentar }\end{array}$ & $\begin{array}{c}\text { Valor } \\
\text { mínimo de } \\
\mathrm{Hg} \text { total } \\
\left(\mu \mathrm{g} \cdot \mathrm{g}^{-1}\right)\end{array}$ & $\begin{array}{c}\text { Valor } \\
\text { máximo de } \\
\mathrm{Hg} \text { total } \\
\left(\mu \mathrm{g} \cdot \mathrm{g}^{-1}\right)\end{array}$ & $\begin{array}{c}\text { Média } \pm \mathrm{DP} \\
\left(\mu \mathrm{g} \cdot \mathrm{g}^{-1}\right)\end{array}$ \\
\hline $\begin{array}{c}\text { Acará } \\
\text { (Geophagus brasiliensis) }\end{array}$ & 24 & iliófago & 0,001 & 0,105 & $0,015 \pm 0,023$ \\
$\begin{array}{c}\text { Tainha } \\
\text { (Mugil sp.) } \\
\begin{array}{c}\text { Robalo } \\
\text { (Centropomus sp.) }\end{array}\end{array}$ & 18 & iliófago & 0,002 & 0,063 & $0,011 \pm 0,014$ \\
$\begin{array}{c}\text { Siri } \\
(\text { Callinectes sp.) } \\
\text { Craca }\end{array}$ & 18 & carnívoro & 0,015 & 0,041 & $0,025 \pm 0,006$ \\
(Balanus sp.) & 10 pools & filtrador & 0,042 & 0,075 & $0,057 \pm 0,016$ \\
\hline \hline
\end{tabular}

Vários autores (Eysink, 1991; Lacerda et al., 1994; Régine et al., 2005) relatam que a assimilação de $\mathrm{Hg}$ pelos peixes está diretamente relacionada com os seus hábitos alimentares e que, dentre estes, os carnívoros se destacam apresentando as maiores concentrações deste metal em um ecossistema.

Os resultados encontrados nas três espécies de peixes confirmam esses dados, visto que, a que apresentou maior teor médio, foi o robalo (Centropomus sp. $-0,025 \mathrm{mg} \cdot \mathrm{g}^{-1}$ ), única espécie predadora utilizada, seguida pelo acará (Geophagus brasiliensis $-0,015 \mathrm{mg} \cdot \mathrm{g}^{1}$ ) e pela tainha (Mugil sp. - 0,011 $\mathrm{mg} \cdot \mathrm{g}^{-1}$ ). Yallouz, Silva e Calixto [S. d.] evidenciaram teores muito superiores em peixes capturados em Itaocara, na região de São Fidélis em 1999. Para o robalo, o teor médio encontrado foi de $0,144 \mathrm{mg} \cdot \mathrm{g}^{-1}$, para a tainha $<0,030 \mathrm{mg} \cdot \mathrm{g}^{-1} \mathrm{e}$, para os exemplares de acará, $0,082 \mathrm{mg} \cdot \mathrm{g}^{-1}$. Entretanto, para os autores, não ficou claro se outros peixes carnívoros apresentariam teores próximos aos limites recomendados se alcançassem peso maior, fato que também foi considerado em nosso estudo, pois os peixes foram cedidos pelos pescadores e não escolhidos pelos autores.

A questão da correlação entre tamanho, peso e/ou idade dos organismos e a concentração de $\mathrm{Hg}$ em seus tecidos, gera controvérsia na literatura. Em nossos resultados, não foi evidenciada tal correlação em nenhuma espécie estudada. Entretanto, Mársico et al. (1999) analisaram amostras de peixes carnívoros (Serrasalmus sp.), oriundos de uma região de garimpo, e observaram uma correlação positiva significativa entre o tamanho dos espécimes analisados e a concentração deste metal $\left(R^{2}=0,91\right)$, resultado este que está de acordo com as considerações realizadas por Lacerda et al. (1994) que afirmam que este fato parece ser bastante típico em áreas de maior poluição ambiental, principalmente áreas de garimpo.

Em áreas não contaminadas, esta correlação, mesmo em espécies consideradas predadoras, nem sempre está presente. Liparisi et al. (2000) correlacionaram o teor de $\mathrm{Hg}$ com o tamanho dos exemplares 
de peixes-espada (Trichurus lepturus) capturados em Itaipu, Niterói, não observando correlação significativa $\left(R^{2}=0,321\right)$. Entretanto, os autores demonstraram uma tendência de acréscimo do valor de $\mathrm{Hg}$ em relação ao tamanho do peixe, o que estaria de acordo com a teoria de bioacumulação de $\mathrm{Hg}$.

Em peixes marinhos, Sellanes et al. (2002) também não observaram uma relação entre a concentração de mercúrio e o tamanho dos peixes. Porém, quando efetuada uma análise por grupo de tamanho, foi encontrada uma correlação positiva e significativa nas amostras com comprimento de até $40 \mathrm{~cm}$. Os autores sugerem que isso poderia estar relacionado com o fato de exemplares mais jovens serem mais vorazes e, nesta fase, assimilarem uma quantidade maior do metal.

Evans, Kathman e Walker (2000) investigaram a concentração de $\mathrm{Hg}$ em siris jovens (Callinectes sapidus) capturados em uma área contaminada com este metal, no Mississipi, Estados Unidos. Em seguida, os animais foram mantidos sob condições laboratoriais, sendo alimentados com peixes possuindo concentrações de $\mathrm{Hg}$ superiores a $1.0 \mathrm{mg} \cdot \mathrm{g}^{-1}$. Os autores observaram nos siris uma assimilação de $\mathrm{Hg}$ de $76 \%$, indicando uma alta eficiência nessa assimilação e uma lenta cinética de excreção. Baseado nisso, os autores concluíram que tais organismos podem biomagnificar o metilHg em cerca de duas a três vezes a concentração na alimentação, ressaltando que a acumulação deste composto mercurial por organismos aquáticos depende da duração e da magnitude de exposição.

Considerando o hábito alimentar dos siris, que são predominantemente carnívoros, se alimentando de restos de peixes e de outros animais, Wakasa et al. (2006) trabalharam com 75 exemplares deste crustáceo oriundos do litoral de Duque de Caxias, Rio de Janeiro. Os autores encontraram teores médios de $\mathrm{Hg}$ total de $0,024 \pm 0,021 \mu \mathrm{g} \cdot \mathrm{g}^{-1}(0,003-0,104)$, não evidenciando correlação entre a concentração de $\mathrm{Hg}$ total, o tamanho e o peso, estando desta forma de acordo com nossos resultados nesta mesma espécie, tanto com relação à correlação, quanto ao teor médio observado na amostragem utilizada $\left(0,023 \mu \mathrm{g} \cdot \mathrm{g}^{-1}\right)$.

As cracas (Balanus sp.) foram escolhidas para este estudo em função dos trabalhos de Ireland (1974) que sugeriu o uso deste organismo marinho como representante da biota que acumula altas concentrações de metais em seus tecidos. Além disso, Niencheski e Baumgarten (2003) descrevem que, pelo fato de serem animais sésseis e com intensa alimentação via filtração da água, são importantes organismos bioindicadores e de relevância para a bioacumulação de metais pesados, visto que possuem importância na alimentação de peixes e crustáceos.

Os dados de Andersen e Depledge (1997), descrevem a concentração de $\mathrm{Hg}$ total e de metilHg em peixes, siris, moluscos e cracas comestíveis do arquipélago de Açores. $\mathrm{O}$ teor médio de $\mathrm{Hg}$ total encontrado na musculatura dos siris foi de $0,725 \mu \mathrm{g} \cdot \mathrm{g}^{-1}(0,412-1,371)$ e nas cracas foi de 0,072 $\mu \mathrm{g} \cdot \mathrm{g}^{-1}(0,036-0,189)$. Destas concentrações, o teor médio de metilHg nos siris foi de $0,660 \mu \mathrm{g} \cdot \mathrm{g}^{-1}(0,375-1,231)$, o que corresponde a $91 \%$ da quantidade de $\mathrm{Hg}$ total; e nas cracas foi de $0,039 \mu \mathrm{g} \cdot \mathrm{g}^{-1}(0,013-0,085)$, equivalente a $54 \%$ do $\mathrm{Hg}$ total. Esta maior porcentagem da forma orgânica do $\mathrm{Hg}$ nas amostras de siris caracteriza o lançamento de $\mathrm{Hg}$ inorgânico neste ecossistema e sugere metilação deste metal. Nas amostras de cracas utilizadas neste estudo, observou-se um teor médio de $\mathrm{Hg}$ total de $0,057 \mu \mathrm{g} \cdot \mathrm{g}^{-1}$, valor este menor que os descritos pelos autores acima citados, mas que, se considerarmos a pouca eficiência do processo de metilação nesta espécie, sugerida pelos mesmos, também podemos considerar que esteja havendo despejo de formas inorgânicas de $\mathrm{Hg}$ neste ecossistema, e de forma contínua. Desta maneira, fica evidente a questão da biomagnificação e de que organismos de tamanho diminuto e sésseis, como as cracas, assimilem $\mathrm{Hg}$ da massa d'água, visto que são filtradores.

Pode-se dizer então que os organismos aquáticos são ótimos indicadores da presença de $\mathrm{Hg}$ e que, de uma forma geral, as concentrações deste metal aumentam à medida que se chega ao final da cadeia alimentar. A este fato pode ser acrescentado que geralmente os teores de $\mathrm{Hg}$ são mais elevados na musculatura do que nas vísceras (Eysink, 1991), aumentando a importância da monitorização do ambiente, visto que a musculatura é a porção do pescado consumida na alimentação.

\section{Conclusão}

Dentre os peixes, foram observados maiores teores de $\mathrm{Hg}$ em exemplares carnívoros (robalo), fato justificado pela relação nítida dos teores de $\mathrm{Hg}$ com o hábito alimentar das espécies analisadas.

Considerando que não foi observada correlação entre $\mathrm{Hg}$ total e o tamanho e/ou peso dos exemplares estudados, pode-se sugerir, embasado em outros estudos, que tal situação esteja relacionada com o grau de contaminação de um ecossistema, pois baixos teores de $\mathrm{Hg}$ total foram encontrados nos representantes filtradores da lagoa Rodrigo de Freitas.

Nos peixes carnívoros, houve uma menor dispersão dos valores de $\mathrm{Hg}$ total, assim como o maior valor médio, resultados estes que ressaltam a importância da monitorização dos níveis de $\mathrm{Hg}$ na biota da lagoa e sugerem a possibilidade de estar ocorrendo metilação no ecossistema estudado.

As cracas foram os organismos que apresentaram o maior teor de $\mathrm{Hg}$ total, podendo sugerir, baseado em dados da literatura, que esteja havendo lançamento de $\mathrm{Hg}$ inorgânico na lagoa Rodrigo de Freitas, ainda que em quantidades pequenas. Seria importante estudar um maior número de cracas, além de verificar a quantidade de metil $\mathrm{Hg}$ e $\mathrm{Hg}$ inorgânico presentes nos tecidos moles destes animais.

Os resultados obtidos também servem de subsídios para uma ação mais efetiva dos órgãos da saúde pública, no que se refere à liberação ou não do consumo de pescado deste ecossistema, e acrescentam conhecimentos para a comunidade científica relacionada com a vigilância sanitária e ciência de alimentos. 


\section{Referências}

ANDERSEN, J.L.; DEPLEDGE, M.H. A survey of total mercury and methylmercury in edible fish and invertebrates from Azorean Waters. Mar. Environ. Research, Grã-Bretanha: Elsevier Science, v. 44, n. 3, p. 331-350, 1997.

AYRES, M.; AYRES JUNIOR, M.; AYRES, D.L.; SANTOS, A. S. Bio Stat 2.0. Aplicações Estatísticas nas áreas de Ciências Biológicas e Médicas. Belém: Sociedade Civil Mamirauá. CNPq. 2000.

CAPPON, C.J.; SMITH, J.C. Chemical form and distribution of mercury and selenium in edible seafood. Journal of Analytical Toxicology, v. 6, jan./fev. 1982.

DEITZ, F.D.; SELL J.L.; BRISTOL, D. Rapid, Sensitive Method for determination of Mercury in a Variety of Biological Samples. Journal of $A O A C$, v. 56, n. 2, p. 378-382, 1973.

EVANS, D.W.; KATHMAN, R.D.; WALKER, W. Trophic accumulation and depuration of mercury by blue crabs (Callinectes sapidus) and pink shrimp (Penaeus duorarum). Mar. Environ. Research, v. 49, n. 5 , p. 419-434, jun. 2000.

EYSINK, G.G.J. A presença de mercúrio nos ecossistemas aquáticos do estado de São Paulo. São Paulo: CETESB - Companhia de Tecnologia de Saneamento Ambiental de São Paulo. 1991, p. 12-28.

FERREIRA JUNIOR, M.G.F.; PLASTINA, B.; LEAL, F.C.; MUROS, F.; FREITAS, P.R.; VALENTIM, F. Indicações para administração sustentável da pesca na lagoa Rodrigo de Freitas, RJ. Revista Brasileira de Medicina Veterinária, v. 25, n. 4, p. 163-166, 2003.

IRELAND, M.P. Variations in the zinc, copper, manganese and lead content of Balanus balanoides in Cardigan Bay, Wales, Ireland. Environmental Pollution, v. 7, n. 1, p. 65-75, 1974.

LACERDA, L.D.; BIDONE, E.D.; GUIMARÃES, A.F.; PFEIFFER, W.C. Mercury concentrations in fish from the Itacaiúnas-Parauapebas river system, Crajás Region, Amazon. An. Acad. Bras. Ci., v. 66, n. 3, p. 373-379, 1994.
LIPARISI, F.; MÁRSICO, E.T.; SANTOS, N.N.; LIMA, F.C. Determinação dos teores de mercúrio em amostras de peixe-espada (Trichurus lepturus), coletadas na praia de Itaipu -Niterói, RJ. Rev. Hig. Alim., v. 14, n. 77, p. 37-39, out. 2000.

MÁRSICO, E.T.; SÃO CLEMENTE, S.C.; SANTOS, N.N.; SILVA, A.J.P. Contaminação de mercúrio em peixes do gênero Serrasalmus coletados no rio Corichão, município de Poconé - MT - Brasil. Rev. Brasil. Ciên. Veterin., Niterói: EdUFF, v. 6, n. 1, p. 18-21, jan./abr. 1999.

NIENCHESKI, L.F.; BAUMGARTEN, M.G. Manual de Procedimentos Metodológicos do Grupo QA\&B (Qualidade Ambiental e Biodiversidade). Protocolo de Análise para Contaminantes. Parte 2 - Determinação de Metais Traço em Organismos. 2003. p. 126-155. Disponível em: www.furg.br/furg/ revistas/atl/at122005.htm. Acesso em: jan 2005.

RÉGINE, M.; GILLES, D.; YANNICK, D.; ALAIN, B. Mercury distribution in fish organs and food regimes: Significant relationships from twelve species collected in French Guiana (Amazonian basin). Science of The Total Environment. Article in press. set. 2005.

SELLANES, A.G.; MARSICO, E.T.; SANTOS, N.N.; SÃO CLEMENTE, S.C.; OLIVEIRA, G.A.; MONTEIRO, A.B.S. Mercúrio em peixes marinhos. Acta Scientiae Veterinariae, v. 30, n. 2, p. 107-112, 2002.

W.H.O. World Health Organization. Environmental Health Criteria 1: "Mercury". Geneva, 1976, 131 p.

WAKASA, Y.S.; SÃO CLEMENTE, S.C.; MÁRSICO, E.T. Contaminação Mercurial em siris e caranguejos da Baía de Guanabara, Rio de Janeiro. Revista Higiene Alimentar, v. 20, n. 138, p. 98-103, 2006.

YALLOUZ, A.V.; SILVA, M.M.; CALIXTO, T.M.P. Avaliação dos teores de $\mathrm{Hg}$ em pescado do trecho Itaocara - São Fidélis, baixo curso do rio Paraíba do Sul. [S. d.] Disponível em: http://www.sbq.org.br/ranteriores/ 23/resumos/1394/ index.html. Acesso em: jan. 2005.

YALLOUZ, A.V.; CALLIXTO, T.M.P. A análise de peixe "in situ" e a possibilidade de intoxicação. Saneam. Amb., n. 90, p. 40-43, set./out. 2002. 